\title{
Geneviève Duchenne - Vincent Dujardjn - Myriam Watthee-Delmotte, Henry Bauchau dans la tourmente $\mathrm{du} X \mathrm{X}^{\mathrm{e}}$ siècle. Configurations historiques et imaginaires
}

\section{Michele Mastroianni}

\section{CpenEdition}

\section{Journals}

\section{Edizione digitale}

URL: http://journals.openedition.org/studifrancesi/7718

DOI: $10.4000 /$ studifrancesi. 7718

ISSN: 2421-5856

\section{Editore}

Rosenberg \& Sellier

\section{Edizione cartacea}

Data di pubblicazione: 1 décembre 2009

Paginazione: 668-669

ISSN: 0039-2944

\section{Notizia bibliografica digitale}

Michele Mastroianni, «Geneviève Duchenne - Vincent Dujardjn - Myriam Watthee-Delmotte, Henry

Bauchau dans la tourmente du xx ${ }^{\mathrm{e}}$ siècle. Configurations historiques et imaginaires», Studi Francesi [Online], 159 (LIII | III) | 2009, online dal 30 novembre 2015, consultato il 09 janvier 2021. URL: http:// journals.openedition.org/studifrancesi/7718; DOI: https://doi.org/10.4000/studifrancesi.7718

Questo documento è stato generato automaticamente il 9 janvier 2021.

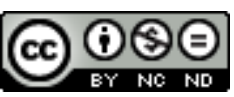

Studi Francesi è distribuita con Licenza Creative Commons Attribuzione - Non commerciale - Non opere derivate 4.0 Internazionale. 


\title{
Geneviève Duchenne - Vincent Dujardjn - Myriam Watthee- Delmotte, Henry Bauchau dans la tourmente $\mathrm{du} \mathrm{XX}^{\mathrm{e}}$ siècle. Configurations historiques et imaginaires
}

\author{
Michele Mastroianni
}

\section{NOTIZIA}

GENEVIÈVE DUCHENNE - VINCENT DUJARDJN - MYRIAM WATTHEE-DELMOTTE, Henry Bauchau dans la tourmente $\mathrm{du} \mathrm{xx}^{\mathrm{e}}$ siècle. Configurations historiques et imaginaires, Bruxelles, Le Cri édition, 2008, pp. 204.

1 Tra gli studiosi che negli anni si sono interessati della scrittura di Henry Bauchau emerge, in particolare, M. Watthee-Delmotte, a cui si deve, fra l'altro, la fondazione di un importante centro all'Università di Louvain, che raccoglie documenti, editi e inediti, tali da offrire materiale di rilievo per l'avanzamento delle ricerche sull'autore belga. Se $\mathrm{M}$. Watthee-Delmotte è nota alla critica per i suoi solidi lavori, che ripercorrono e si configurano all'interno di una prospettiva di ricerca axée sull'immaginario e sulla complessità elaborativa dell'opera bauchauliana, secondo una riflessione critica eminentemente letteraria, ora Watthe-Delmotte dà alle stampe, in collaborazione con due storici come G. Duchenne e V. Dujardin, anch'essi noti al pubblico per il rigore delle loro ricerche, un libro che, pur presentandosi come manualetto divulgativo, è consigliabile per la ricchezza dei dati e, soprattutto, per le informazioni bibliografiche e per la fitta presenza di lettere e di inediti ivi pubblicati integralmente o in parte, che insieme alla presentazione dei tre curatori, non solo smentisce la veste di manualetto sotto cui si presenta, ma chiarisce l'intento scientifico degli autori, facendo inoltre luce 
sulla sua validità critica, tale per cui questo saggio appare consigliabile anche a chi non si fosse ancora occupato degli scritti di Henry Bauchau.

Dunque, due gli assi di indagine: da un lato una ricerca storica che muove da documenti d'archivio fino a questo momento sconosciuti, dall'altro una speculazione letteraria che parte anche da numerosi manoscritti inediti del periodo giovanile bauchauliano, conservati appunto nei Fonds Henry Bauchau dell'Università Cattolica di Louvain. Due prospettive di ricerca che si incontrano nel vivo dell'indagine, apportando l'un l'altra materiale di interesse per lo storico e per il letterato, ripercorrendo una figura poetica che emerge dalla sua profondità e con una certa completezza di informazioni, proprio attraverso l'intreccio delle due dinamiche esistenziali: storica e poetica. Così, dai numerosi dati che inquadrano storicamente l'autore all'interno di una stagione esistenziale cruciale per la sua formazione e maturazione, stagione in cui egli si distingue per militanza cattolica attiva e impegnata (siamo negli anni TrentaQuaranta), ma che lo segna anche per delusione e sofferenza profonde, si passa al 1958, data alla quale Bauchau lega la sua prima vera comparsa sulla scena letteraria come poeta. In questa seconda sezione viene ripercorsa per tappe la vita dell'autore, attraverso una indagine sensibile, in parallelo, al riflesso del personale $\mathrm{e}$ dell'esistenziale nell'opera letteraria, proprio nel tentativo di sottolineare l'importanza della imprescindibilità dell'elemento storico dal dato letterario. In tale prospettiva dunque è condotto lo studio di questa seconda sezione in bilico fra esistenziale letterario e storico esistenziale. Una sezione strutturata intorno a momenti e a luoghi determinanti per Henry. Parigi, poi la Svizzera con il suo coinvolgimento nell'Istituto Montesano che egli fonda, poi ancora Parigi, dopo il fallimento di quello stesso Istituto, ma questa volta una Parigi dove egli si sperimenterà come psicoterapeuta e come analista di giovani psicotici. Così, attraverso i dati biografici, esistenziali e poetici Watthee-Delmotte, all'interno del quadro storico tracciato nella prima sezione del volumetto, ricorda l'importanza della psicanalisi per Bauchau, il ruolo determinante esercitato dall'incontro con Blanche Reverchon Jouve e l'apertura vitale alla scrittura attraverso la poesia e la prosa. Apprezzabile anche per la bibliografia, che insiste sull'aspetto storico della vita dell'autore, ricordando contributi scientifici di primaria importanza, questo libro merita una segnalazione per il materiale informativo che offre, oltre che per la coerenza di dati e di struttura, nel limite, tuttavia, di quella relativa brevitas che si impone in saggi i cui contenuti si rivolgono anche ad un pubblico di non addetti ai lavori. 